\title{
AN ELECTROMECHANICAL MICROPOLAR PERIDYNAMIC MODEL FOR ISOTROPIC AND ORTHOTROPIC MATERIALS
}

\author{
VITO DIANA ${ }^{1}$ AND VALTER CARVELLI ${ }^{1}$ \\ ${ }^{1}$ Department ABC, Politecnico di Milano, Piazza Leonardo da Vinci 32, Milano, 20133, Italy \\ vito.diana@polimi.it
}

Key words: Peridynamics, Multiphysics, Fracture, Micropolar model, Orthotropy

\begin{abstract}
A micropolar peridynamic model for in-plane electro-mechanical behavior of isotropic and orthotropic solids is presented. The analytical implicit formulation of the electrical part of the model is based on the definition of a proper microelectrical energy function and a specific electrical inelastic deformation parameter. A compatibility condition and a constitutive relationship has been derived and thus the electrical stiffness operator has been obtained. The electrical formulation is then coupled with a mechanical micropolar peridynamic formulation that accounts for full orthotropy and isotropy as special case. A distinctive aspect of the formulation is the use of continuous trigonometric functions, for the mechanical and electrical bond properties with respect to the principal material axes. The obtained unified model is capable to predict the mechanical response and the electrical conduction of elastic brittle materials taking into account the influence of cracks and other defects along with mechanical and/or electrical orthotropy. The proposed model has been applied to predict the electric field potential in isotropic and orthotropic square laminae, and to simulate a coupled electromechanical problem.
\end{abstract}

\section{INTRODUCTION}

Peridynamics is a non-local theory of mechanics [1] in which continuity requirements on the displacement field are removed [2]. The originally proposed theory, referred to as bond-based PD (BBPD), is a central force model. Therefore, Cauchy Relations hold $[3,4,5]$ and for isotropic materials Poisson's ratio is restricted to $v=1 / 4$ for three-dimensional (3D) and plane strain, while $v=1 / 3$ for plane stress. Silling et al. [6] introduced later a more general formulation, namely state-based (SBPD) in which the pairwise force between two particles depends on the deformations of all nodes within their neighborhood. Other efforts along these lines include the non-ordinary state-based model (NOSB) [7, 8], the conjugated bond-pair-based PD formulation [9], and the Gerstle's non-local lattice beam model [10]. Recently, a generalized continuum micropolar model for isotropic and orthotropic materials, inspired by Voigt's studies on crystals [11], has also been presented [12,13] and validated for isotropic and full orthotropic materials.

The nature of partial differential equations allows Peridynamics to be considered as general nonlocal continuum theory, and thus to be extended also to other physical fields. The peridynamics equations have been applied by Gerstle et al. [14] to the one-dimensional electromigration, by using a framework which allows four coupled physical processes to be modeled simultaneously: mechanical deformation, heat transfer, electrical potential distribution, and vacancy diffusion. Bobaru and Duangpanya proposed 
a one dimensional peridynamic heat conduction equation, and solved the $2 \mathrm{D}$ peridynamic transient heat conduction problem in bodies with evolving discontinuities following a bond-based approach $[15,16]$ and using a direct implementation of the peridynamic balance equations. An analytical derivation of a state-based peridynamic model of heat diffusion was presented by Oterkus et al. [17]. The same authors proposed later a coupled peridynamic thermo-mechanic model starting from thermodynamic considerations [18], and using explicit schemes to approximate the solutions of the peridynamic equations. Other applications of peridynamics for thermo-mechanics or other multi-physics problems can be found in $[19,20]$.

In the context of electrical conduction and coupled electro-mechanical models, Prakash and Seidel [2] developed a classical bond-based formulation for the study of the piezoresistive response of carbon nanotube reinforced polymer nanocomposite materials. The authors applied then their formulation to model the strain and damage sensing in nanocomposite bonded explosive materials [21, 22]. In this paper, the generalized micropolar peridynamic (MPPD) formulation [12, 13, 23], is extended to electrical conduction and electro-mechanical problems in orthotropic materials. The electrical peridynamic model for orthotropic materials is analytically derived and is then coupled with the orthotropic mechamical micropolar formulation that does not have restrintions in the number of independent material constants. A distinctive aspect of the model, is that each bond connecting two particles can be mechanistically regarded as an assemblage of three mechanical springs (two translational and one rotational ) and a electrical conductive ligament. Another distinctive aspect of the formulation is the use of continuous trigonometric functions, for the orientation of the mechanical and electrical bond properties with respect to the principal material axes. The obtained unified model is capable to predict the elastic response and the electrical conduction of elastic brittle materials taking into accou
defects along with mechanical and/or electrical orthotropy. The accur
assessed by predicting the electric field potential in isotropic and
simulation of a coupled electromechanical problem.

2 ORTHOTROPIC ELECTRICAL CONDUCTION MODEL
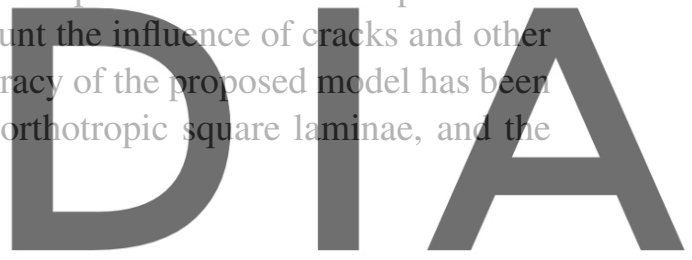

Register for free at https//www.scipedia.com to download the version without the watermark The balance equation of the electrical conduction for a continuum whose material points interact through pairwise potentials, e.g. peridynamic continuum, for any infinitesimal material particle at position $\mathbf{X}$ and time $t$ is $[23,2]$

$$
\int_{H_{\mathbf{X}}} f_{I}\left(\phi^{\prime}-\phi, \mathbf{X}^{\prime}-\mathbf{X}\right) d V_{\mathbf{X}^{\prime}}+\omega(\mathbf{X}, t)=c_{e} \dot{\phi}(\mathbf{X}, t) \quad \text { for } \quad \mathbf{X} \in \Omega,
$$

where $\Omega$ is the domain occupied by the body, whereas $\mathbf{X}^{\prime}-\mathbf{X}=\xi$ is the relative position between the material points $\mathbf{X}$ and $\mathbf{X}^{\prime}$. In the case of non-linearly coupled mechanical-electrical (or thermal) problems, $f_{I}$ could be also function of $\mathbf{u}^{\prime}-\mathbf{u}=\eta$, which defines the relative displacement between $\mathbf{X}$ and $\mathbf{X}^{\prime}$. The integral is defined over a region $H_{\mathbf{X}}$ called horizon of radius $\delta$ or family of $\mathbf{X}$ [1]. $\phi$ is the particle's electric potential, $c_{e}$ is the electrical capacitance of the material, $\omega$ is the net current per unit volume at $\mathbf{X}$. The kernel or integrand function $f_{I}$ has the dimensions of an electric current per unit volume squared $[15,2]$. In the proposed electrical conduction model, we introduce the parameter $e=-\frac{\left(\phi^{\prime}-\phi\right)}{|\xi|}$ that can be considered as the equivalent of a ligament deformation measure in elasticity. It is worth underlying that for the general case of electro-mechanical problems, the bond electric field is 
along the line connecting the two particles in the deformed configuration. The compatibility equation relating the particles primary field $\phi$ to the magnitude of the bond electrical field $e$ is

$$
e=\{b\}^{T}\{\phi\} \longrightarrow\{b\}^{T}=-\frac{1}{|\xi|}\{-1 \quad 1\}
$$

The bond constitutive equation characterizing the electrical model is derived by a specific pair inelastic potential function for electrical conduction

$$
\mathrm{w}_{e}=\frac{k_{e} e^{2}}{2}=\frac{k_{e}\left(\phi^{\prime}-\phi\right)^{2}}{2|\xi|^{2}}
$$

where $k_{e}$ is the ligament conductivity parameter, namely microconductivity of the electrical model, having the dimension of an electric conductivity per unit volume.

The electrical pairwise interaction (namely electrical response function) is then obtained as the first variation of the potential function $\mathrm{w}_{e}$

$$
f_{I}=\frac{\partial \mathrm{w}_{\mathrm{e}}}{\partial \eta_{\phi}}=\frac{k_{e} e}{|\xi|}=\frac{k_{e}\left(\phi^{\prime}-\phi\right)}{|\xi|^{2}}
$$

\section{where $\eta_{\phi}=\left(\phi^{\prime}-\phi\right)$. The bond micro-current density vector can be then defined as}

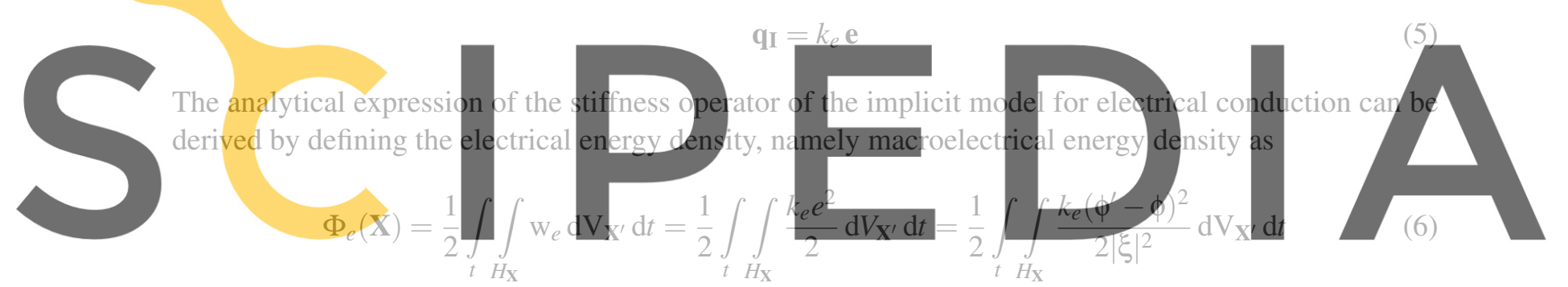

\section{Register for free at https/fwww scipedia.comato download the version without the watermark}

external electrical work per unit time of the interaction between particles $i$ and $j$ can be written as

$$
\frac{1}{2}\{\phi\}^{T} \frac{1}{2} \alpha \Delta V_{i} \Delta V_{j}\{b\} k_{e}\{b\}^{T}\{\phi\}=\frac{1}{2}\{\phi\}^{T}\left\{f_{I}^{*}\right\}
$$

where

$$
\{\phi\}^{T}=\left\{\begin{array}{ll}
\phi^{i} & \phi^{j}
\end{array}\right\} ; \quad\left\{f_{I}^{*}\right\}^{T}=\left\{\begin{array}{ll}
f_{I}^{i} & f_{I}^{j}
\end{array}\right\}
$$

being $\left\{f_{I}^{*}\right\}$ the array of the external actions. The equivalent stiffness operator is

$$
[K]_{e}=\alpha \Delta V_{i} \Delta V_{j}\{b\} k_{e}\{b\}^{T}
$$

where $\alpha$ is the volume correction coefficient that takes into account the partial neighbor intersections. 


\subsection{Micro-conductivities}

In the classical theory of homogenized elastic orthotropic materials, the electrical conductivity typically exhibits a directional dependent variation, whereas the current density is not necessarily parallel to the electric field. The relationship between the orthogonal components of the current density and the electrical field in the principal material system, inclined at angle $\zeta$ with respect to the horizontal, is given by

$$
\mathbf{q}_{\mathbf{I}}=\mathbf{D}_{\mathbf{e}} \mathbf{e} \longrightarrow\left\{\begin{array}{l}
q_{I_{1}} \\
q_{I_{2}}
\end{array}\right\}=\left[\begin{array}{cc}
K_{e_{11}} & 0 \\
0 & K_{e_{22}}
\end{array}\right]\left\{\begin{array}{l}
e_{1} \\
e_{2}
\end{array}\right\}
$$

In Eq. 10, $\mathbf{D}_{\mathbf{e}}$ is symmetric, second-rank tensors called electrical conductivity tensor of components $K_{e_{i i}}$. Assuming here for simplicity that $\zeta=0$, thus considering an arbitrary coordinate system $x y$ rotated by $\psi$ with respect to the horizontal, the off-axis conductivity $K_{e_{x x}}$ can be written as function of the direction defined by angle $\psi$, and of the two material constants defined in Eq. (10) as

$$
K_{e_{x x}}(\psi)=K_{e_{11}} \cos ^{2} \psi+K_{\mathrm{e}_{22}} \sin ^{2} \psi
$$

By analogy with the phenomenological classical description of anisotropic conductivity, we can assume for instance that the ligament microconductivity $k_{e}$ is function of $\psi$ (the bond inclination angle) as $K_{e_{x x}}$ in Eq 11, namely

$$
k_{e}(\psi)=k_{e_{1}} \cos ^{2} \psi+k_{\mathrm{e}_{2}} \sin ^{2} \psi
$$

where $k_{e_{1}}$ and $k_{e_{2}}$ are the peridynamic axial micromoduli along the principal material axes. Thus, two independent microcond uct orthotropic solid. The relationship between orthotropic continuum par (namely the macroelectric general homogeneous eleetric field, which is

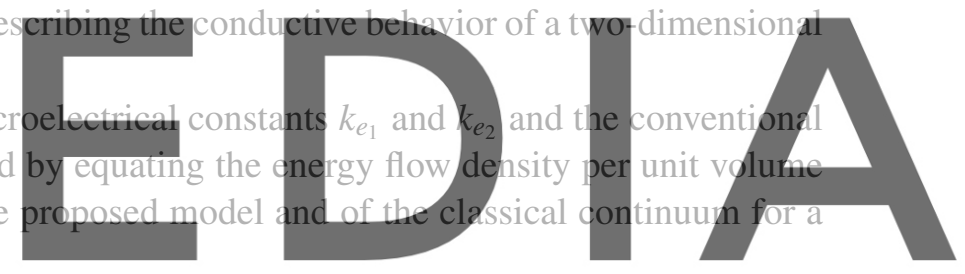

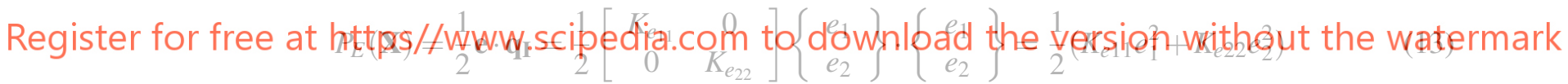

The corresponding quantity of the proposed model is instead given by the general integral

$$
P_{e}(\mathbf{X})=\frac{1}{2} \int_{H_{\mathbf{X}}} \mathrm{w}_{e} \mathrm{dV}_{\mathbf{X}^{\prime}}=\frac{1}{2} \int_{H_{\mathbf{X}}} \frac{k_{e}(\psi) e^{2}(\psi)}{2} \mathrm{~d} V_{\mathbf{X}^{\prime}}=\frac{1}{2} \int_{H_{\mathbf{X}}} \frac{k_{e}(\psi)\left(e_{1} \cos \psi+\mathrm{e}_{2} \sin \psi\right)^{2}}{2} \mathrm{~d} V_{\mathbf{X}^{\prime}}
$$

in which, assuming $k_{e}(\psi)$ described by Eq. 12, leads to

$$
P_{e}(\mathbf{X})=\frac{1}{32}\left[e_{1}^{2}\left(3 k_{e 1}+k_{e 2}\right)+e_{2}^{2}\left(k_{e 1}+3 k_{e 2}\right)\right] \delta^{2} \pi \mathrm{t}
$$

Hence, comparing Eqs. 13 and 15, and collecting the terms multiplying the same electric field components, we obtain the system of two equations $W_{E}(\mathbf{X})_{i}=\Phi(\mathbf{X})_{i}, i=1 \ldots 2$

$$
\left\{\begin{array}{l}
\delta^{2}\left(3 k_{e 1}+k_{e 2}\right) \pi \mathrm{t}=16 \mathrm{~K}_{\mathrm{e} 11} \\
\delta^{2}\left(k_{e 1}+3 k_{e 2}\right) \pi \mathrm{t}=16 \mathrm{~K}_{\mathrm{e} 22}
\end{array}\right.
$$


which provides

$$
k_{e 1}=\frac{2\left(3 K_{e 11}-K_{e 22}\right)}{\pi \mathrm{t} \delta^{2}} ; \quad k_{e 2}=\frac{2\left(3 K_{e 22}-K_{e 11}\right)}{\pi \mathrm{t} \delta^{2}}
$$

For the case of isotropy we have $K_{e 11}=K_{e 22}=K_{e}$. Hence $k_{e 1}=k_{e 2}=k_{e}$, where

$$
k_{e}=\frac{4 K_{e}}{\pi \mathrm{t} \delta^{2}}
$$

which is the same expression of $k_{e}$ for isotropic materials derived in [15, 23].

When principal material directions are not aligned with the horizontal and vertical directions, (being $\zeta \neq 0$ ), Eq. 12 can be rewritten as

$$
k_{e}(\psi)=k_{e_{1}} \cos ^{2}(\psi-\zeta)+k_{\mathrm{e}_{2}} \sin ^{2}(\psi-\zeta)
$$

It should be noted that other laws of variation of the bond inelastic modulus with respect to the ligament inclination angle can be assumed, allowing to model different degrees of anisotropy. For instance, a more general description of $k_{e}(\psi)$ as function of the ligament orientation could be

$$
k_{e}(\psi)=k_{e_{2}}+\left(k_{e_{1}}-k_{e_{2}}\right) \cos ^{n} \psi
$$

where $n=2 N$, with $N \subseteq \mathbb{N}$ (Eq. 12 is got assuming $n=2$ in Eq. 20)

3 ORTHOTROPIC ELECTRO-MECHANICAL MODEL
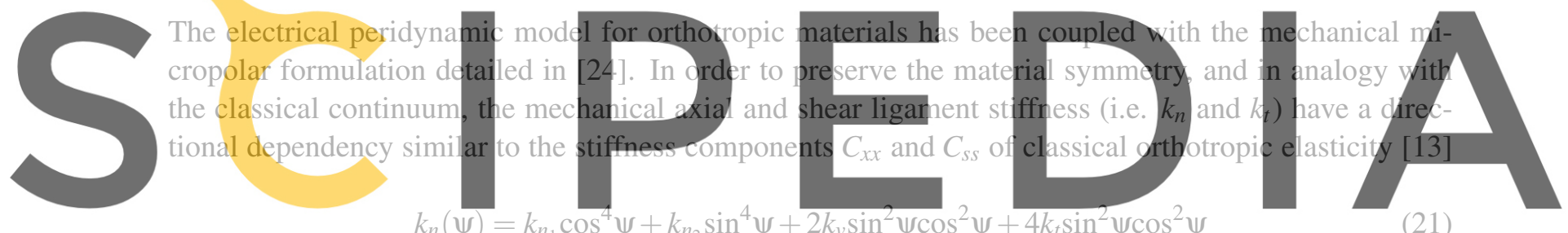

$$
k_{n}(\psi)=k_{n_{1}} \cos ^{4} \psi+k_{n_{2}} \sin ^{4} \psi+2 k_{v} \sin ^{2} \psi \cos ^{2} \psi+4 k_{t} \sin ^{2} \psi \cos ^{2} \psi
$$

where $k_{n_{1}}$ and $k_{n_{2}}$ are the axial micromoduli along principal material axes, $k_{t_{1}}=k_{t_{2}}=k_{t}$ is the shear micromodulus along the principal material axis and $k_{v}$ is related to the ratio between $k_{t}(\psi)$ and $k_{n}(\psi)$ in each ligament direction. The orthotropic microelastic moduli, $k_{n_{1}}, k_{n_{2}}, k_{v}$ and $k_{t}$ can be written as function of the classical elastic constants that define in-plane orthotropy, following an approach based on energetic equivalence between the present model and classical continuum

$$
\begin{array}{rlrl}
k_{n_{1}} & =\frac{12\left(C_{11}-C_{66}\right)}{\pi t \delta^{3}} ; & k_{n_{2}}=\frac{12\left(C_{22}-C_{66}\right)}{\pi t \delta^{3}} \\
k_{v}=\frac{12\left(3 C_{12}-C_{66}\right)}{\pi t \delta^{3}} ; & k_{t}=\frac{3\left(8 C_{66}-C_{11}-C_{22}-2 C_{12}\right)}{\pi t \delta^{3}}
\end{array}
$$

In the case of isotropy, $C_{11}=C_{22}$ and $C_{12}=C_{11}-2 C_{66}$. Eqs. (23) and (24) provide two independent microelastic constants in terms of $C_{11}$ and $C_{66}$ only, as

$$
k_{n_{1}}=k_{n_{2}}=k_{n}=\frac{12\left(C_{11}-C_{66}\right)}{\pi t \delta^{3}} ; \quad k_{t}=\frac{12\left(3 C_{66}-C_{11}\right)}{\pi t \delta^{3}} ; \quad k_{v}=k_{n}-2 k_{t}
$$


The ligament global stiffness is

$$
[K]=\left[[K]_{m}+[K]_{e}\right]
$$

where $[K]_{m}$ is the bond stiffness operator characterizing the mechanical model. A distinctive distinctive aspect of the proposed electromechanical formulation is the use of continuous trigonometric functions, being the mechanical and electrical bond properties continuous functions of its orientation with respect to the principal material axes. Therefore $[K]$ depends on the ligament orientation angle.

\section{APPLICATIONS}

The accuracy of the proposed formulation is illustrated to predict the electric field potential in a square lamina $(l=40 \mathrm{~mm})$ with a central hole of radius $r=5 \mathrm{~mm}$. The applied potential difference at the top and bottom horizontal edges is $\Delta \phi=20 \mathrm{~V}$, whereas the domain is discretized using a regular grid spacing $\Delta x=0.5 \mathrm{~mm}$ resulting in a model of 6084 particles. Two materials are considered: an isotropic material (MAT 1) with electrical conductivity $K_{e}$ and an orthotropic material (MAT 2) with $K_{e_{1}} / K_{e_{2}}=0.45$ and $\zeta=\pi / 4$. The mechanical model has been extensively validated in linear elasticity $[12,13]$, therefore
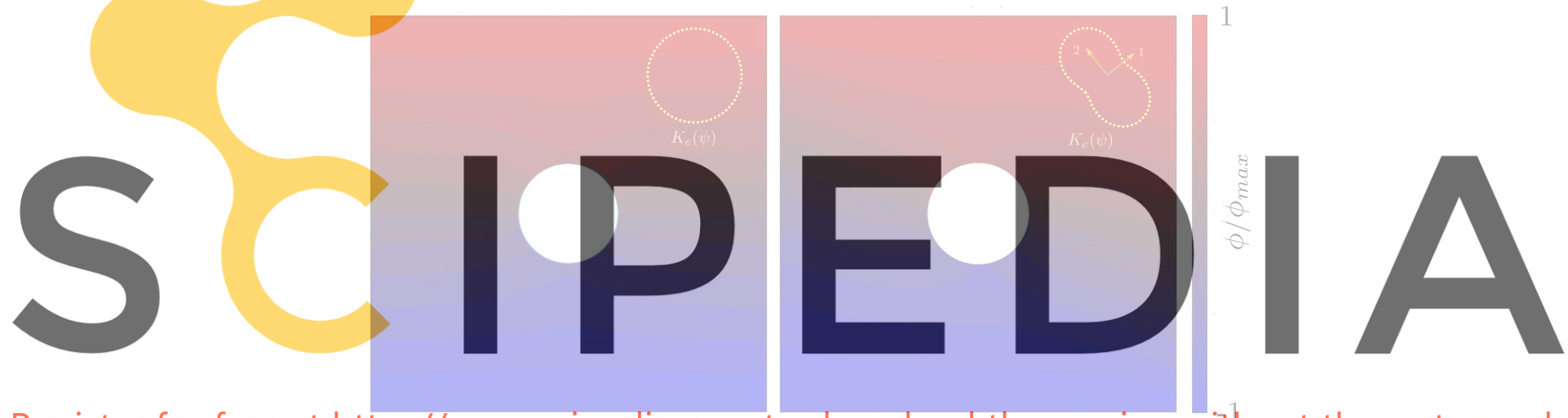

Figure 1: Square laminae with central hole and made of isotropic MAT 1 and orthotropic MAT $2(\zeta=\pi / 4)$ :

Normalized electric potential distribution by the proposed model.

the attention is here on the electrical part of the model, hence no mechanical boundary conditions are considered. Results obtained using the present peridynamic formulation are reported in Fig. 1.

In the second application, a preliminary assessment of the numerical performance of the proposed formulation in the presence of strong discontinuities such as cracks, is carried out studying the problem of electrical conduction of a rectangular lamina with an insulated crack. The layout of the problem is shown in Fig. 2, where $a=400 \mathrm{~mm}$ and $b=200 \mathrm{~mm}$, and the assigned length of the crack $l$ is progressively increased from 0 to $0.9 \mathrm{~b}$. The lamina is of unit thickness, electrical conductivity $K_{e_{1}} / K_{e_{2}}=0.45$ and an electrical potential difference $\Delta \phi=2 \mathrm{~V}$ applied to the top and bottom edges. The discretized model is composed by 3200 particles (regular grid of $80 \times 40$ particles with $\delta=0.0375 a$ ). The pre-existing crack is introduced by breaking all of the interactions passing through the crack surfaces at the beginning of the simulation. For each assigned crack length, the electrical field potential is computed with the proposed model, hence the net current $I$ flowing into the lamina is calculated by summing up the electric reactions 

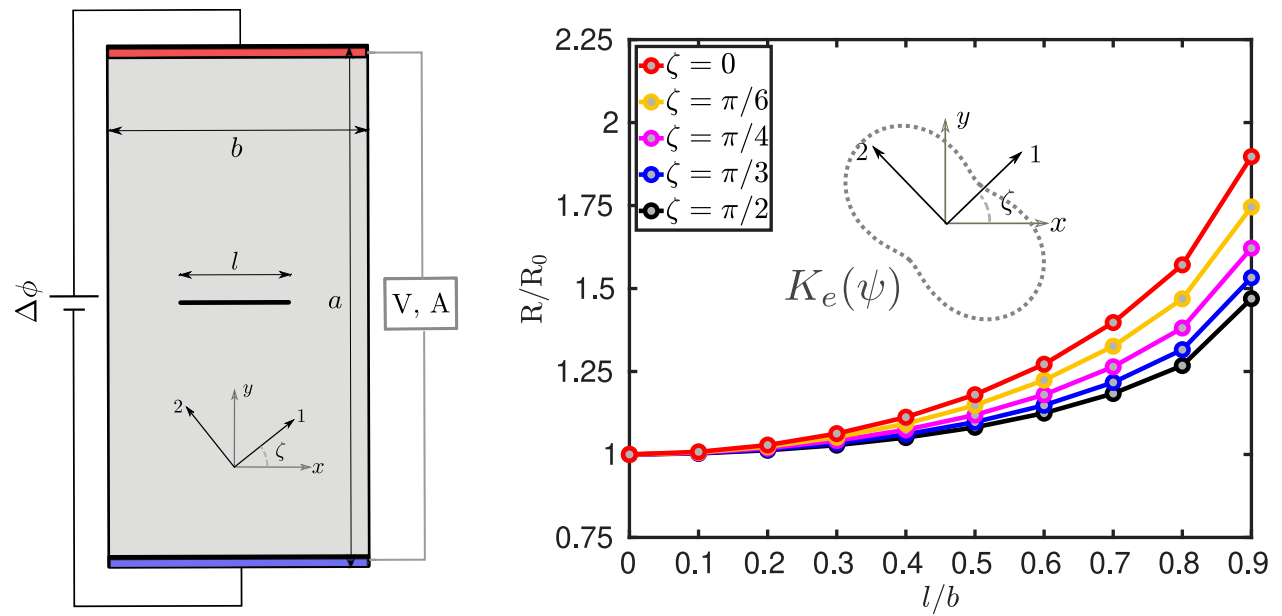

Figure 2: Electrical conduction in a orthotropic rectangular lamina with a horizontal crack: (left) Layout of the problem; (right) Variation of the electrical resistance as function of the propagating crack length for different $\zeta$. The electrical resistances are normalized respect to the resistance $R_{0}$ of the uncracked lamina.

(concentrated currents) corresponding to the nodes in which the negative electric potential is applied. In this way, applying Ohm's law

$$
R=\Delta \phi / I
$$

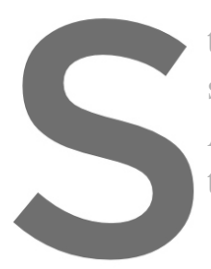

the electrical resistance $R$ ses are reported in Fig.

As final application, the p

trical conduction for dama

In particular, the results
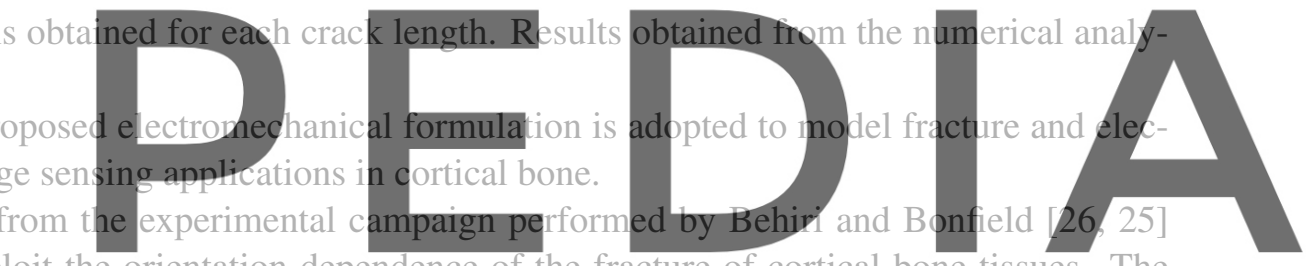

are here considered to exploit the orientation dependence of the fracture of cortical bone tissues. The

Register fectromechanical setup under consideration is detailed in Fig. 3 where the longitudinal and radial axes

Register for free at https/ Wwww scipedia, com to downlo thad the version without the watermark

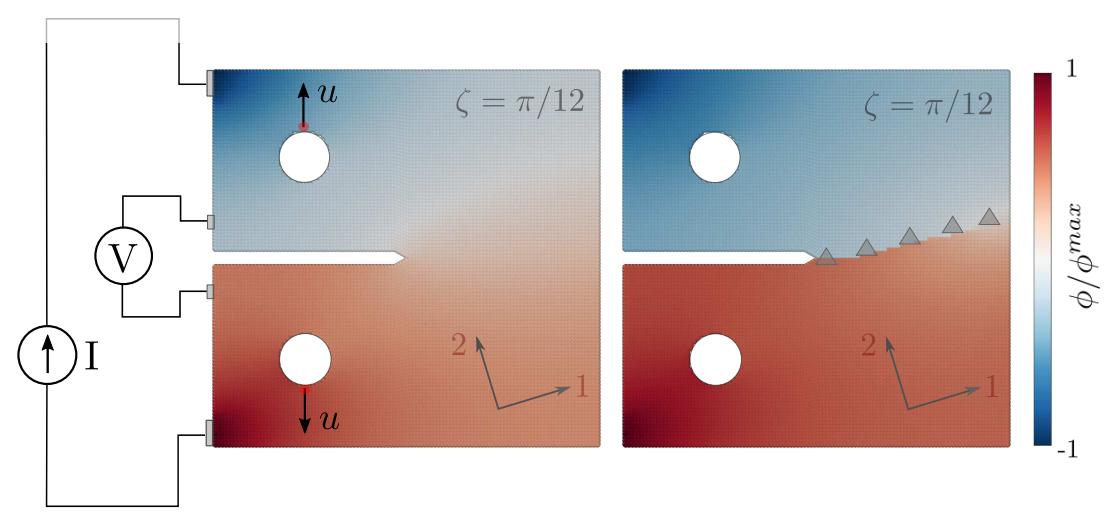

Figure 3: Fracture profiles and electric field potential in cortical bone specimen with $\zeta=\pi / 12$. Layout of the problem and normalized electric field potential of uncracked specimens (left). Normalized electric field potential of cracked specimens with indication of the experimental crack profiles (triangles) from [25, 26] (right). 


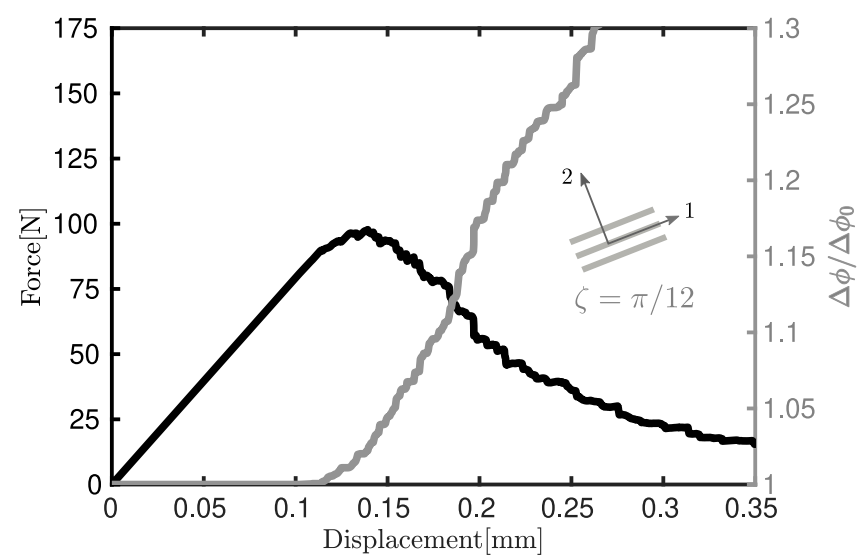

Figure 4: Potential drop and force-displacement relationship corresponding to cortical bone specimens with material reference system inclined at $\zeta=\pi / 12$.

different angle of anisotropy $\zeta$ as performed by Behiri and Bonfield [25] has been simulated using the proposed formulation and employing an energetic failure criterion [24] accounting here for non-uniform toughness. The mechanical and electrical conduction models have been then coupled in the hypothesis of small deformations [23] to study the effect of evolving discontinuities on the conductive behavior of cortical bone tissues. Orthotropic elastic constants and failure properties reported in [27], are adopted in this study. Regarding instead the electrical conductivity properties of cortical bone tissues, a ratio $K_{e_{1}} / K_{e_{2}} \simeq 3$ as reported in Reddy and Sahd [28] is used. Results obtained considering $\zeta=\pi / 12$ are
reported in Figs. 3 and 4 . In particular, Fig. 3 shows the agreement of the crack path predicted using the
proposed model and the experimental craek profile. Normalized variation of the potential output as func-
tion of the applied total displacement corresponding to cortical bone specimen with material reference
system inclined at $\zeta=\pi / 12$ is instead shown in Fig. 4 .

\section{Register för freedGt hitpsy\%www.scipedia.com to download the version without the watermark}

In this paper an analytical peridynamic formulation for in-plane electrical conduction and fracture in isotropic and orthotropic materials has been proposed. By defining a proper microelectrical energy function and an electrical inelastic deformation parameter, the stiffness operator of the non-local electrical conduction has been derived. The electrical peridynamic model has been then coupled with an in-plane mechanical orthotropic micropolar formulation characterized by four independent elastic parameters. The proposed model has been applied to predict the electric field potential in isotropic and orthotropic laminae, and the influence of crack propagation in the effective electrical conductive properties of a cortical bone tissue.

\section{REFERENCES}

[1] Silling, S.A., Reformulation of elasticity theory for discontinuities and long-range forces. Journal of the Mechanics and Physics of Solids, 48(1):175-209, 2000.

[2] Prakash, N. and Seidel, G. D., Electromechanical peridynamics modeling of piezoresistive response 
of carbon nanotube nanocomposites. Computational Materials Science, 113:154 - 170, 2016.

[3] Navier, C.L.M.H. , Mémoire sur les lois de l'équilibre et du mouvement des corps solides élastiques. Mémoire lu a l'Académie royale des sciences, 6:375-384, 1827.

[4] Cauchy, A., Mémoire sur les systèmes isotropes de points matériels. Mémoires de l'Académie des sciences, t. XXII:351-386, 1850.

[5] Poisson, S.D., Mémoire sur les équations générales de l'équilibre et le du mouvement des corps solides élastiques et des fluides. Journal de l'École polytechnique, 13(20):1-174, 1831.

[6] Silling, S.A., Epton, M., Weckner, O., Xu, J. and Askari, E., Peridynamic states and constitutive modeling. Journal of Elasticity, 88(2):151-184, 2007.

[7] Warren, T. L., Silling, S.A., Askari, A., Weckner, O., Epton, M., and Xu, J., A non-ordinary statebased peridynamic method to model solid material deformation and fracture. International Journal of Solids and Structures, 46(5):1186 - 1195, 2009.

[8] Li, P., Hao, Z.M., and Zhen, W.Q., A stabilized non-ordinary state-based peridynamic model. Computer Methods in Applied Mechanics and Engineering, 339:262 - 280, 2018.

[9] Wang, Y., Zhou, X., Wang, Y., and Shou, Y., A 3-d conjugated bond-pair-based peridynamic formulation for initiation and propagation of cracks in brittle solids. International Journal of Solids and Structures, 134:89-115, 2018.

[10] Gerstle, W., Sau, N., and Silling, S.A., Peridynamic modeling of concrete structures. Nuclear

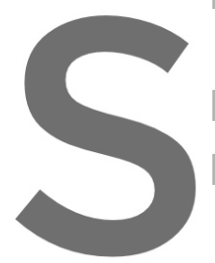
Engineering and Des

[11] Voigt, W., Lehrbuch der Kr

[12] Diana, V., and Casolo, S., A bond-bresed mi ity: Elasticity, failure Structures, 160:201-231, 2019
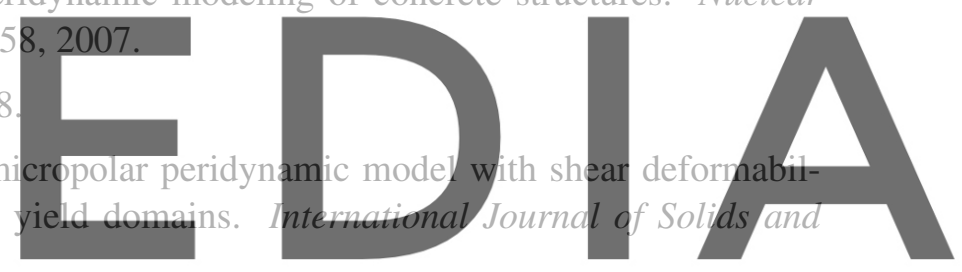

Register for free at Kittps Gasolo. S. A full orthotropic micropolarperidynamic formulation for linearly elastic

[14] Gerstle, W., Silling, S.A., Read, D., Tewary, V., and Lehoucq, R., Peridynamic simulation of electromigration. Tech Science Press, 8(2):75-92, 2008.

[15] Bobaru, F., and Duangpanya, M., The peridynamic formulation for transient heat conduction. International Journal of Heat and Mass Transfer, 53(19):4047 - 4059, 2010.

[16] Bobaru, F., and Duangpanya, M., A peridynamic formulation for transient heat conduction in bodies with evolving discontinuities. Journal of Computational Physics, 231(7):2764 - 2785, 2012.

[17] Oterkus, S., Madenci, E., and Agwai, A., Peridynamic thermal diffusion. Journal of Computational Physics, 265:71 - 96, 2014.

[18] Oterkus, S., Madenci, E., and Agwai, A., Fully coupled peridynamic thermomechanics. Journal of the Mechanics and Physics of Solids, 64:1 - 23, 2014.

[19] Bazazzadeh, S., Mossaiby, F., and Shojaei, A., An adaptive thermo-mechanical peridynamic model for fracture analysis in ceramics. Engineering Fracture Mechanics, 223:106708, 2020. 
[20] Zhang, Y., Pan, G., Zhang, Y., and Haeri, S., A multi-physics peridynamics-dem-ib-clbm framework for the prediction of erosive impact of solid particles in viscous fluids. Computer Methods in Applied Mechanics and Engineering, 352:675 - 690, 2019.

[21] Prakash, N., and Seidel, G.D., Computational electromechanical peridynamics modeling of strain and damage sensing in nanocomposite bonded explosive materials (ncbx). Engineering Fracture Mechanics, 177:180 - 202, 2017.

[22] Prakash, N., and Seidel, G.D., Effects of microscale damage evolution on piezoresistive sensing in nanocomposite bonded explosives under dynamic loading via electromechanical peridynamics. Modelling and Simulation in Materials Science and Engineering, 26(1):015003, dec 2017.

[23] Diana. V., and Carvelli, V., An electromechanical micropolar peridynamic model. Computer Methods in Applied Mechanics and Engineering, 365:112998, 2020.

[24] Diana, V., and Ballarini, R., Crack kinking in isotropic and orthotropic micropolar peridynamic solids. International Journal of Solids and Structures, 196-197:76-98, 2020.

[25] Bonfield, W., and Behiri, J.C., Fracture toughness of natural composites with reference to cortical bone. Composite Materials Series, 6(C):615-635, 1989.

[26] Bonfield, W., and Behiri, J.C., Orientation dependence of the fracture mechanics of cortical bone. Journal of Biomechanics, 22(8):863-872, 1989.

[27] Ghajari, M., Iannucci, L., and Curtis, P., A peridynamic material model for the analysis of dynamic

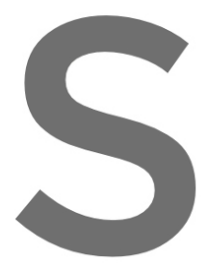
crack propagation in $276: 431-452,20$

[28] Reddy, G.N., and Saha, S., quency. IEEE Transactions
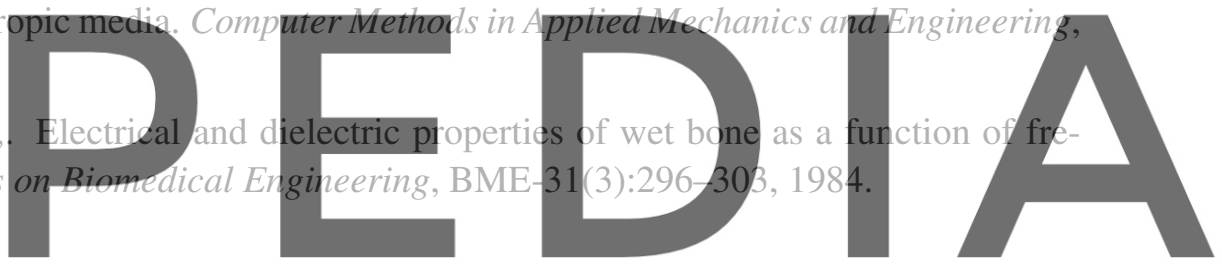

Register for free at https//www.scipedia.com to download the version without the watermark 